\title{
Omental reinforcement of the thoracic esophagogastric anastomosis: An analysis of leak and reintervention rates in patients undergoing planned and salvage esophagectomy
}

\author{
Boris Sepesi, MD, Stephen G. Swisher, MD, Garrett L. Walsh, MD, Arlene Correa, PhD, \\ Reza J. Mehran, MD, David Rice, MD, Jack Roth, MD, Ara Vaporciyan, MD, and Wayne L. Hofstetter, MD
}

Objective: An uncontained thoracic anastomotic leak may cause severe morbidity or mortality. Thoracic transposition of an omental flap along with the gastric conduit may decrease leak incidence, severity, or need for reoperations after esophagectomy.

Methods: We identified 607 patients who underwent esophagectomy with thoracic anastomosis between Janu-
ary 2001 and August 2011 . All patients were studied for leak postoperatively. Four leak grades were defined,
ranging from radiographic leak to conduit loss. Univariate and multivariate analyses were performed to identify
variables associated with anastomotic leak.

Results: Omental reinforcement was used in 215 of 607 patients (35\%). Anastomotic leak occurred in 51 of 607 patients $(8.4 \%)$. Patients with omentum had a significantly lower (odds ratio $[\mathrm{OR}], 0.4 ; P=.014$ ) anastomotic leak rate $(4.7 \%)$ compared with patients without omentum $(10.5 \%)$. Salvage resections were performed in 69 patients and $23(32 \%)$ received omentum; the leak rate with omentum was $4.6 \%$ compared with $15 \%$ without (OR, 0.27; $P=.24$ ). Patients undergoing planned esophagectomy with omentum had a significantly lower leak rate than patients without omentum $(4.7 \%$ vs $9.8 \%)(\mathrm{OR}, 0.43 ; P=.04)$. Reoperations to rescue an anastomotic leak (Grade 3) was less common with omentum $(\mathrm{OR}, 0.26 ; P=.024)$. Multivariate analysis identified omental reinforcement $(\mathrm{OR}, 0.45 ; P=.034)$ and surgeon $(\mathrm{OR}, 3.4 ; P=.001)$ as variables predictive of an anastomotic leak.

Conclusions: Omental reinforcement of thoracic esophagogastric anastomoses decreases overall leak rate and need for reoperation. We recommend pedicled omental transposition to reinforce all thoracic anastomoses. Endoscopic evaluation of significant anastomotic leaks is still warranted. (J Thorac Cardiovasc Surg 2012;144:1146-51)

Earn CME credits at

http://cme.ctsnetjournals.org

Leak of a thoracic esophagogastric anastomosis may be associated with significant morbidity, prolonged hospital stay, and the potential need for reoperation. Thoracic anastomotic leak has also been associated with higher short-term (eg, 90 days) postoperative mortality compared with patients without anastomotic leak. ${ }^{1}$ Those patients who successfully recover from this complication have a 4-fold increased risk of eating difficulties, including odynophagia or dysphagia. ${ }^{2}$

From the Department of Thoracic and Cardiovascular Surgery, MD Anderson Cancer Center, Houston, Tex.

Disclosures: Authors have nothing to disclose with regard to commercial support.

Read at the 92nd Annual Meeting of The American Association for Thoracic Surgery, San Francisco, California, April 28-May 2, 2012.

Received for publication April 26, 2012; revisions received July 11, 2012; accepted for publication July 30, 2012; available ahead of print Aug 30, 2012.

Address for reprints: Wayne L. Hofstetter, MD, Department of Thoracic and Cardiovascular Surgery, University of Texas MD Anderson Cancer Center, 1515 Holcombe Blvd, Houston, TX 77030 (E-mail: whofstetter@mdanderson.org). $0022-5223 / \$ 36.00$

Copyright $($ c 2012 by The American Association for Thoracic Surgery http://dx.doi.org/10.1016/j.jtcvs.2012.07.085
During the past decade, early recognition and appropriate management of thoracic anastomotic leaks has significantly decreased leak-associated mortality, historically cited between $50 \%$ and $70 \%$. Our group previously reported a leak-associated mortality of 3.3\% despite increased use of neoadjuvant chemoradiation therapy. ${ }^{3}$ However, these improvements in outcome were derived at the expense of early operative reintervention when leaks were identified.

Enveloping the thoracic anastomosis with a pedicled omental flap has been previously demonstrated to decrease the leak rate. ${ }^{4-6}$ However, widespread acceptance of this additional operative step is lacking for various reasons. Given its potential merits, we have progressively embraced the practice of omental reinforcement of thoracic esophagogastrostomy. The aim of our study was to evaluate the anastomotic leak rate and the need for reintervention in patients who underwent esophagectomy with purely thoracic esophagogastric anastomosis with or without pedicled omental flap reinforcement.

\section{METHODS}

\section{Patients}

The study population consisted of 607 consecutive patients selected from a population of 946 patients who underwent esophagectomy at MD 


\section{Abbreviation and Acronym \\ $\mathrm{POD}=$ postoperative day}

Anderson Cancer Center between January 2001 and August 2011; 7 surgeons contributed data to the study. The use of omentum for the reinforcement of the thoracic anastomosis was progressively adopted over time (Figure 1). All patients who underwent esophageal resection for esophageal carcinoma with esophagogastric anastomosis reconstruction within the chest were potentially included in our study. Patients undergoing salvage esophagectomy, defined as resection $>3$ months after failed definitive chemoradiation, were included, as were the patients who received neoadjuvant multimodality therapy followed by planned resection (Planned Group). Excluded patients included those with cervical esophagogastric anastomoses $(\mathrm{n}=316)$, esophagojejunal anastomoses $(\mathrm{n}=5)$, and redo or emergent operations $(\mathrm{n}=0)$. Patients who had a thoracic anastomosis primarily reinforced with a muscle flap, pericardial fat pad, or pleural flap $(\mathrm{n}=18)$ were also excluded.

\section{Operation and Perioperative Course}

All patients underwent esophageal resection and restoration of the intestinal continuity with thoracic esophagogastric anastomosis. The anastomosis was created using either a Collard-type linear stapled technique or an intraluminal technique with size 25 or $29 \mathrm{~mm}$ circular stapler. Omental reinforcement was used in 215 patients $(35 \%)$. The omental pedicle was created during the abdominal portion of the operation by mobilizing the omentum off of the transverse colon. The omental pedicle was based on 2 to 4 perforators from the right gastroepiploic artery. Therefore, only a portion of the omentum is used as a pedicled flap, which was transposed into the chest along with the gastric conduit. Once in the chest it was positioned like a bun around a hot dog, medial to lateral, to envelop the entire anastomosis as well as the gastric staple line.

Postoperatively, patients' conditions were managed on a monitored, dedicated thoracic ward unless a clear indication mandated intensive care unit admission. Enteral nutrition via jejunostomy tube began between Postoperative Days (POD) 1 through 3; nasogastric tube was discontinued on POD 5. Unless there was indication for earlier intervention, all patients underwent a routine radiographic study to evaluate for anastomotic leak between POD 7 and 14. A gastrograffin followed by a thin barium swallow was used to evaluate the leak. The presence or absence of leak was recorded in all patients. The decision regarding the management of an anastomotic leak was guided by the radiographic findings, overall clinical status of the patient, and the discretion of an attending surgeon.

\section{Data Collection}

Clinical and operative records were retrospectively reviewed from a prospectively maintained database. All patients with evidence of anastomotic leak were classified into 1 of 4 grades of leak severity, similar to the previously established Clavien classification of surgical complications, which categorizes the complications based on the invasiveness and necessity for intervention. ${ }^{7}$ Four grades of anastomotic leak were defined ranging from contained radiographic leak to the loss of gastric conduit (Table 1). Other study variables included age, sex, body mass index, omentum, histology, tumor location, type of esophagectomy, clinical and pathologic stage, surgeon, year of operation, American Society of Anesthesiologists classification, salvage or planned esophagectomy, preoperative radiation therapy, and the dose of radiation. The surgeon variable was introduced into the analysis as a dichotomous variable (higher or lower leak) to account for unmeasured variables related to surgical technique. Modified en bloc esophagectomy was defined as D2 abdominal and thoracic lymphadenectomy (Levels 7, 8, 9) with ligation of the thoracic duct and resection of the posterior mediastinal tissue medial to the azygous vein, which was left in situ.

\section{Statistical Analysis}

The primary aim of our study was to evaluate the influence of omental reinforcement on the incidence of thoracic esophagogastric anastomotic leak. We further assessed the classification and methods of reintervention for anastomotic leak in patients with and without omental reinforcement. Statistical analyses to evaluate variables associated with anastomotic leak included Pearson $\chi^{2}$ test, Fisher exact test, and univariate logistic regression. Variables with $P<.25$ on univariate analysis were further tested with a backward stepwise multivariable logistic regression (Wald Statistic) to ascertain the influence of omental reinforcement. The interaction between variables was assessed.

The study received approval of the MD Anderson Cancer Center Institutional Review Board. A waiver of consent was granted for this retrospective data review.

\section{RESULTS}

Table 2 describes the clinicopathologic characteristics of the entire population and of groups with and without anastomotic reinforcement. In a predominantly male population (median age 61 years; range, 23 to 83 years) with body mass index $>25(70 \%)$, the most common tumor histology was distal adenocarcinoma (92\%). Clinical stage 2 and 3 disease was the operative indication in $83 \%$ of patients, and a majority of patients $(84 \%)$ received neoadjuvant therapy. Omentum was used as an anastomotic reinforcement in 215 patients $(35 \%)$. This technique was used more commonly (78\%) during modified en bloc esophageal resection and less commonly during minimally invasive approach $(23 \%)$.

Anastomotic leak occurred in a total of $8.4 \%$ of all included patients. The incidence of leak for a thoracic esophagogastric anastomosis was significantly lower (odds ratio [OR], $0.4 ; P=.014$ ) in patients with pedicled omental flap reinforcement $4.7 \%$ compared with those without omental reinforcement $(10.5 \%)$. Although not statistically significant, the absolute difference in overall leak rate was most prominent in salvage esophagectomy patients, where observed anastomotic leak rate in patients with omentum was $4.6 \%$ versus $15 \%$ in patients without omentum (OR, $0.272 ; P=.24)$. Anastomotic leak in patients with planned esophagectomy who underwent omental buttress was $4.7 \%$ compared with $10 \%$ without omentum (OR, $0.44 ; P=.04)$.

With regard to leak severity, there was no significant difference in Grade 1 and 2 leaks between the groups with and without omentum. However, there was a significantly lower need for operative rescue of leaks (Grade 3 ) in patients with omental pedicle (1.4\%) compared with patients without omentum (5.1\%) (OR, 0.26; $P=.024)$ (Table 3). There was no difference in Grade 4 leak between the groups.

Table 4 illustrates the statistical analyses. Univariate analysis identified the use of omental pedicle to be associated with favorably low incidence of anastomotic leak (OR, 0.42; $P=.016$ ). Distal esophageal tumor location was close to reaching significance as a protective variable. (OR, 0.33; $P=.054$ ). Leak rate was also significantly associated with surgeon (OR, $0.45 ; P=.008)$. The year of surgery had no influence on either leak rate $(P=.65)$ 


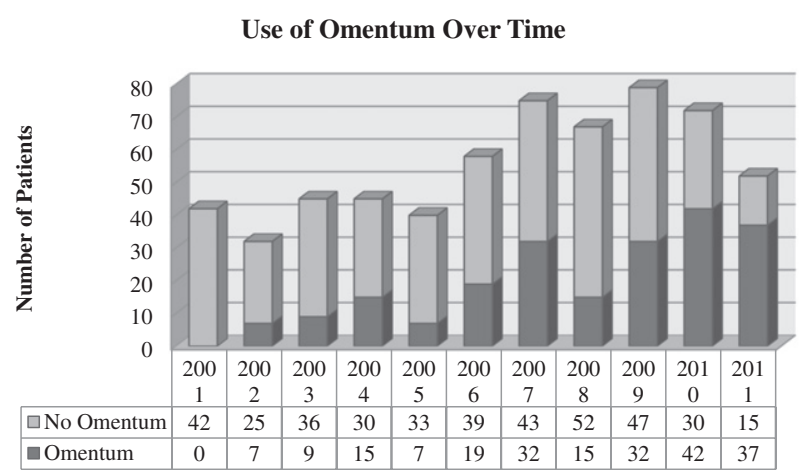

FIGURE 1. Frequency of use of omental pedicle reinforcement of the thoracic esophagogastrostomy over time.

or leak grade $(P=.904)$. Multivariate logistic regression identified omental reinforcement of the thoracic anastomosis $(\mathrm{OR}, 0.2 ; P=.001)$ and surgeon $(\mathrm{OR}, 3.4 ; P=.001)$ as independent predictors of leak rate. A separate multivariable analysis $(\mathrm{n}=540)$, excluding patients undergoing minimally invasive esophagectomy, also identified omentum $(\mathrm{OR}, 0.3 ; P=.008)$ and surgeon $(\mathrm{OR}, 2.9 ; P=.006)$ as independent predictors of outcome. In the multivariable analysis we assessed the interaction between variables; however, no statistical significance was found.

Postoperatively, we observed significantly less $(P=.014)$ major pulmonary complications (eg, pneumonia, acute respiratory distress syndrome, and reintubation) in the group with omentum. There was no difference in cardiovascular $(P=.273)$ or wound $(P=.324)$ complications or hospital readmission $(P=.324)$ rates.

Thirty-day mortality was $2.6 \%(\mathrm{n}=16), 90$-day mortality was $4 \%(\mathrm{n}=24)$; neither 30-day $(P=.718)$ nor 90 -day $(P=.507)$ mortality were significantly different between the groups. Leak-associated mortality was $5.8 \%$ in patients without omentum and $1.9 \%$ in patients with omentum $(P=1.0)$.

\section{DISCUSSION}

To our knowledge, ours is the largest study analyzing the influence of omentum on intrathoracic esophagogastric

TABLE 1. Classification and definition of leak grade

\begin{tabular}{lc}
\hline Leak grade & Definition \\
\hline 1 & Contained radiographic leak only not requiring any invasive \\
& intervention. Allowed therapeutic regimen included: \\
& nothing per os, antibiotics, antipyretics, analgesics. \\
& Leak requiring either endoscopic or percutaneous invasive \\
& intervention, such as endoluminal stent or percutaneous \\
& drain placement under radiographic guidance. \\
& Anastomotic leak necessitating operative intervention (ie, \\
& thoracotomy) to control mediastinal sepsis. Anastomotic \\
& dehiscence was repaired in this scenario and reinforced \\
& with muscle flap. Gastrointestinal continuity was rescued \\
& with reoperation. \\
& Anastomotic dehiscence secondary to conduit necrosis \\
& requiring conduit resection with esophageal diversion. \\
\hline
\end{tabular}

TABLE 2. Demographics of total population and patients with and without omentum

\begin{tabular}{|c|c|c|c|c|c|c|c|}
\hline Population & $\begin{array}{c}\text { All } \\
\text { patients }\end{array}$ & $(\%)$ & $\begin{array}{c}\text { No } \\
\text { omentum }\end{array}$ & (\%) & Omentum & & $\begin{array}{c}P \\
\text { value }\end{array}$ \\
\hline & 607 & & 392 & 65 & 215 & 35 & \\
\hline Length of stay (d) & & & 11 & & 9 & & .001 \\
\hline \multicolumn{8}{|l|}{ Age (y) } \\
\hline Median & 61 & & & & & & \\
\hline Range & $23-83$ & & & & & & \\
\hline \multicolumn{8}{|l|}{ Sex } \\
\hline Man & 534 & 89 & 346 & 65 & 188 & 35 & .765 \\
\hline Woman & 73 & 12 & 46 & 63 & 27 & 37 & \\
\hline \multicolumn{8}{|l|}{ Histology } \\
\hline Adenocarcinoma & 561 & 92 & 358 & 64 & 203 & 36 & .290 \\
\hline SCC & 30 & 5 & 21 & 70 & 9 & 30 & \\
\hline Other histology & 16 & 3 & 13 & 81 & 3 & 19 & \\
\hline \multicolumn{8}{|l|}{ Tumor location } \\
\hline Proximal & 19 & 3 & 15 & 79 & 4 & 21 & .183 \\
\hline Distal & 588 & 97 & 377 & 64 & 211 & 36 & \\
\hline \multicolumn{8}{|l|}{ Esophagectomy } \\
\hline $\begin{array}{c}\text { Minimally } \\
\text { invasive }\end{array}$ & 67 & 10 & 52 & 77 & 15 & 23 & .018 \\
\hline Ivor Lewis & 540 & 90 & 340 & 63 & 200 & 37 & \\
\hline Planned & 538 & 89 & 346 & 64 & 192 & 36 & .700 \\
\hline Salvage & 69 & 11 & 46 & 67 & 23 & 33 & \\
\hline $\begin{array}{l}\text { Modified En } \\
\text { Bloc }\end{array}$ & 102 & 17 & 22 & 22 & 79 & 78 & .001 \\
\hline \multicolumn{8}{|l|}{ Other therapy } \\
\hline Neoadjuvant & 509 & 84 & 329 & 65 & 180 & 35 & .947 \\
\hline No neoadjuvant & 98 & 16 & 63 & 64 & 35 & 36 & \\
\hline \multicolumn{8}{|l|}{ Body mass index } \\
\hline$<25$ & 181 & 30 & 110 & 61 & 71 & 39 & .201 \\
\hline$>25$ & 426 & 70 & 282 & 66 & 144 & 34 & \\
\hline \multicolumn{8}{|l|}{ Clinical stage } \\
\hline 0 & 9 & 1.5 & 7 & 78 & 2 & 22 & .152 \\
\hline 1 & 65 & 10.5 & 37 & 57 & 28 & 43 & \\
\hline 2 & 234 & 38 & 144 & 62 & 90 & 38 & \\
\hline 3 & 272 & 45 & 184 & 66 & 88 & 32 & \\
\hline 4 & 26 & 4 & 20 & 77 & 6 & 23 & \\
\hline \multicolumn{8}{|l|}{ Pathologic stage } \\
\hline 0 & 126 & 21 & 72 & 57 & 54 & 43 & .126 \\
\hline 1 & 108 & 18 & 67 & 62 & 41 & 38 & \\
\hline 2 & 233 & 38 & 161 & 69 & 72 & 31 & \\
\hline 3 & 128 & 21 & 82 & 64 & 46 & 36 & \\
\hline 4 & 12 & 2 & 10 & 83 & 2 & 17 & \\
\hline \multicolumn{8}{|l|}{ ASA classification } \\
\hline $1-2$ & 129 & 21 & 94 & 73 & 35 & 27 & .027 \\
\hline $3-4$ & 478 & 79 & 298 & 62 & 180 & 38 & \\
\hline \multicolumn{8}{|l|}{ Radiation therapy } \\
\hline$\leq 50.4$ & 486 & 80 & 319 & 66 & 167 & 34 & .192 \\
\hline$>50.4$ & 16 & 3 & 7 & 44 & 9 & 56 & \\
\hline None & 102 & 17 & 65 & 64 & 37 & 36 & \\
\hline Unknown & 3 & 0.004 & & & & & \\
\hline
\end{tabular}

anastomotic leak rate. Our data demonstrates significantly decreased anastomotic leak rate when the anastomosis is reinforced with omental pedicle flap; the use of omentum was 
TABLE 3. Leak and leak grade in omentum and no omentum groups

\begin{tabular}{lccccccc}
\hline & $\begin{array}{c}\text { All } \\
\text { patients }\end{array}$ & $\begin{array}{c}\text { No } \\
\text { omentum }\end{array}$ & $\begin{array}{c}\text { Omentum } \\
\text { Population }\end{array}$ & \multicolumn{5}{c}{$\begin{array}{c}\boldsymbol{P} \\
\text { value }\end{array}$} & cOR & $\mathbf{9 5 \%}$ CI \\
\hline Total leak & $51(8.4)$ & $41(10.5)$ & $10(4.7)$ & .014 & 0.4 & $0.2-0.85$ \\
No leak & $556(91.6)$ & $351(89.5)$ & $205(95.3)$ & & & \\
Leak Grade 1 & $15(2.5)$ & $13(5)$ & $2(1)$ & .09 & 0.27 & $0.06-1.2$ \\
Leak Grade 2 & $7(1.2)$ & $6(1.5)$ & $1(0.5)$ & .43 & 0.30 & $0.03-2.51$ \\
Leak Grade 3 & $23(4)$ & $20(5.1)$ & $3(1.4)$ & .024 & 0.26 & $0.07-0.89$ \\
Leak Grade 4 & $6(1)$ & $2(0.5)$ & $4(1.9)$ & .192 & 3.7 & $0.67-20.4$ \\
\hline
\end{tabular}

cOR, Crude odds ratio; $C I$, confidence interval.

also an independent predictor of lower leak incidence. In addition, when omentum was used we observed a significantly decreased need to reoperate on patients with severe leak when the esophagogastric continuity could be salvaged (Grade 3). Predictably, when gastric conduit necrosis occurred, removal of the conduit with esophageal diversion was indicated regardless of the omental pedicle use.

Surgeons have recognized the natural tendency of the omentum to wall off perforations and to isolate infectious processes for decades. The omentum has been shown to possess anti-inflammatory, immunologic, angiogenic, and drainage functions. These inherent characteristics may provide a suitable healing environment and perhaps compensate for unfavorable factors in anastomotic healing such as relative ischemia or radiation effect. ${ }^{8}$ Reinforcing

TABLE 4. Univariate and multivariate logistic regression analysis*

\begin{tabular}{|c|c|c|c|c|c|c|}
\hline \multirow[b]{2}{*}{ Variable } & \multicolumn{3}{|c|}{ Univariate } & \multicolumn{3}{|c|}{ Multivariate } \\
\hline & $\begin{array}{c}P \\
\text { value }\end{array}$ & OR & CI & $\begin{array}{c}P \\
\text { value }\end{array}$ & OR & CI \\
\hline Omentum & .016 & 0.418 & $0.20-0.85$ & .001 & 0.2 & $0.12-0.56$ \\
\hline Surgeon & .008 & 0.45 & $0.25-0.81$ & .001 & 3.4 & $1.8-6.4$ \\
\hline Distal tumor & .054 & 0.33 & $0.10-1.02$ & .068 & 0.33 & $0.10-1.08$ \\
\hline Sex & .952 & 0.97 & $0.4-2.36$ & & & \\
\hline Age & .897 & 0.99 & $0.97-1.02$ & & & \\
\hline MIE & .001 & 3.59 & $1.82-7.06$ & .25 & 1.65 & $0.7-3.89$ \\
\hline En bloc & .89 & 1.005 & $0.49-2.21$ & & & \\
\hline $\mathrm{BMI} \geq 25$ & .48 & 1.26 & $0.65-2.43$ & & & \\
\hline cStage 1 & .856 & 0.814 & $0.08-7.65$ & & & \\
\hline cStage 2 & .626 & 0.587 & $0.06-4.9$ & & & \\
\hline cStage 3 & .845 & 0.81 & $0.09-6.74$ & & & \\
\hline cStage 4 & .972 & 1.04 & $0.09-11.52$ & & & \\
\hline pStage 1 & .914 & 0.95 & $0.37-2.38$ & & & \\
\hline pStage 2 & .929 & 1.03 & $0.48-2.22$ & & & \\
\hline pStage 3 & .791 & 0.88 & $0.36-2.16$ & & & \\
\hline pStage 4 & .999 & 0.000 & 0.000 & & & \\
\hline ASA 3 and 4 & .17 & 1.75 & $0.77-4.02$ & .232 & 1.66 & $0.72-3.86$ \\
\hline $\mathrm{XRT} \geq 50.4 \mathrm{~Gy}$ & .89 & 1.05 & $0.48-2.32$ & & & \\
\hline Salvage & .313 & 0.66 & $0.29-1.47$ & & & \\
\hline Surgery year & .65 & 1.02 & $0.92-1.12$ & & & \\
\hline
\end{tabular}

an esophagogastrostomy with a well-vascularized omentum is therefore intuitively sound. In fact 2 randomized trials have demonstrated a significantly decreased leak rate of esophagogastric anastomosis reinforced with omental pedicle; despite this data anastomotic reinforcement with the omentum is not uniformly practiced.

Our data corroborates that of others. In 1987 Zhang and colleagues $^{9}$ reported on 128 esophagectomy cases performed between 1979 and 1984. A pedicled omentum flap to reinforce the esophagogastric anastomosis was used in 100 of 128 patients. Those authors did not observe any postoperative anastomotic leaks in patients with omental wrapping of the anastomosis. Two patients required reoperation, 1 for intrathoracic colon herniation attributed to inadequate omental mobilization of the transverse colon and 1 for an intrathoracic abscess raising a question of a potential anastomotic leak. In both cases, the authors noted the omentum to be densely adherent to the anastomosis. ${ }^{9}$

Since that study, 2 prospective randomized trials have demonstrated the benefit of omental wrapping in decreasing anastomotic leaks. In 2006, Bhat and colleagues ${ }^{4}$ reported on 184 patients equally randomized to undergo omental wrapping of the esophagogastric anastomosis. Both transthoracic and transhiatal esophagectomy techniques were used. The anastomotic leak rate with omental buttress was $3.4 \%$ compared with $14.4 \%$ without omentum $(P=.005)$. There were no complications reported related to omental pedicle mobilization, and no mortality difference was observed. ${ }^{4}$ Those authors recommended omental pedicle transposition to decrease anastomotic leak rate; however, they also indicated that omental transposition should not be used to compensate for a technically inadequate anastomosis or compromised conduit viability. Dai and colleagues ${ }^{5}$ conducted the second randomized trial on 255 patients, 127 of whom received anastomotic omental reinforcement. Again, both transthoracic and transhiatal resection techniques were used. Anastomotic leak rate was $1 \%$ (with omentum) versus 6\% (without omentum) $(P=.05)$. Anastomotic stricture was also lower in the omental group $(6 \%$ vs $16 \% ; P<.05)$.

These data support the use of omental reinforcement of esophagogastrostomy, and its beneficial effect in decreasing anastomotic leak rate, consequences of which may extend beyond the perioperative complications. ${ }^{5}$ Our data are in agreement with previously published data, in addition to our study being the largest of its kind in the Western world demonstrating the benefit of omental reinforcement on purely thoracic anastomoses.

The limitations of our study include biases associated with the retrospective study design. Unaccounted and difficult-to-measure variables such as differences in individual operative technique or a clustering effect can influence the outcome of the statistical analyses. These differences are accounted for in the surgeon variable. 
Interestingly, time was not a significant variable contributing to the end point. Also, although the surgeon variable remained significant on the multivariate analysis, which takes into account the entire study period, all surgeons observed a lower anastomotic leak incidence when they converted to utilizing omentum to reinforce thoracic anastomoses.

We have found the use of omental pedicle reinforcement of the thoracic anastomosis beneficial. Mobilization of a portion of the omentum from the transverse colon and creation of the pedicle adds approximately 20 minutes to the operative time, and we have not observed any additional morbidity from omental mobilization. On the contrary, the overall rate of pulmonary complications in patients who received omental transfer was lower compared with patients without omental transfer. None of our tracked perioperative complications were increased by the additional operative step. Leak-associated mortality was low in both groups with and without omentum.

\section{CONCLUSIONS}

We recommend routine use of the omentum to reinforce intrathoracic esophagogastric anastomoses (Level A-B evidence, Class I-IIa recommendation). However, the omentum should be viewed as an adjunct to a well-vascularized interpolate and technically sound anastomosis, and not as a substitute for an inadequate conduit. Likewise, judicious postoperative care and appropriate workup for anastomotic leak is still warranted for all patients undergoing esophagectomy.

\section{References}

1. Rutegård M, Lagergren P, Rouvelas I, Lagergren J. Intrathoracic anastomotic leakage and mortality after esophageal cancer resection: a population-based study. Ann Surg Oncol. 2012;19:99-103.

2. van der Schaaf M, Lagergren J, Lagergren P. Persisting symptoms after intrathoracic anastomotic leak following oesophagectomy for cancer. Br J Surg. 2012;99:95-9.

3. Martin LW, Swisher SG, Hofstetter W, Correa AM, Mehran RJ, Rice DC, et al. Intrathoracic leaks following esophagectomy are no longer associated with increased mortality. Ann Surg. 2005;242:392-9; discussion 399-402.

4. Bhat MA, Dar MA, Lone GN, Dar AM. Use of pedicled omentum in the esophagogastric anastomosis for prevention of anastomotic leak. Ann Thorac Surg. 2006; $82: 1857-62$

5. Dai JG, Zhang ZY, Min JX, Huang XB, Wang JS. Wrapping of the omental pedicle flap around anastomosis after esophagectomy for esophageal cancer. Surgery. 2011;149:404-10.

6. Yuan Y, Hu Y, Xie TP, Zhao YF. Omentoplasty for preventing anastomotic leaks after esophagogastrostomy. Surgery. 2011;149:853-4.

7. Dindo D, Demartines N, Clavien P-A. FACS classification of surgical complications: a new proposal with evaluation in a cohort of 6336 patients and results of a survey. Ann Surg. 2004;240:205-13.

8. Kitano M. Omentoplasty in thoracic surgery. Gen Thorac Cardiovasc Surg. 2008; 56:483-9.

9. Zhang K, Yang YH. Use of pedicled omentum in oesophagogastric anastomosis: an analysis of 100 cases. Ann R Coll Surg Engl. 1987;69:209-11.

\section{Discussion}

Dr Gail Darling (Toronto, Ontario, Canada). Dr Sepesi and colleagues address an important topic in esophageal surgery, that of the anastomotic leak. They report an $8.4 \%$ overall leak rate for intrathoracic anastomoses and demonstrate reduced leak rates in patients who were buttressed with omentum.

I have several questions. First, how should we incorporate the results of your study into practice? Should we do it for all patients? In your study, how were patients selected for omental buttress? Was it simply surgeon preference? Was it an intraoperative decision where the surgeon was concerned about the status of the conduit or the anastomosis, or were there preoperative factors that influenced the decision to use omentum?

Dr Sepesi. We believe that it is safe to use the omentum on all patients. As our complication analysis demonstrated, there was no increase in complications. It takes approximately 20 minutes of extra time to mobilize the omentum of the transverse colon and to create the pedicle based on 3 perforating vessels off of the right gastroepiploic artery. We believe that it may decrease the leak rate in all patients.

Dr Darling. How did you select your patients for omental buttress?

Dr Sepesi. The use of omentum has increased over time in our practice. Initially, we used the omentum in patients undergoing salvage esophagectomy who had higher anastomotic leak rates. We expanded that to patients undergoing planned esophagectomy in an attempt to completely eliminate anastomotic leaks.

Dr Darling. It is hard to evaluate the results of an intervention over a prolonged period of time. Do you think we should conduct a randomized trial?

Dr Sepesi. There have been 2 randomized trials performed outside the United States. Both included patients with intrathoracic as well as cervical anastomoses. Both studies demonstrated favorably low leak rate when the omentum was used. Our study is the only retrospective study focusing purely on intrathoracic anastomosis. Properly designed randomized trials with adequate statistical power to demonstrate a significant difference in leak rate would likely require enrollment of several hundreds of patients. Considering the relatively innocuous nature of the omental anastomotic reinforcement, a randomized trial may not be necessary.

Dr Darling. I have a bit of trouble with the retrospective application of the Clavien classification. You define a Grade 3 complication as 1 that requires operative intervention, yet your conclusion was that the buttressing reduced the need for operative intervention. Can you explain how you retrospectively applied that classification?

Dr Sepesi. We retrospectively analyzed the leak rate in the 2 groups, 1 with the omentum and 1 without the omentum. We assigned each leak a severity grade based on the need for invasive intervention. We observed that when the omentum was used there was an overall lower leak rate for all leak grades combined; however, the most prominent difference was that we did not have to reoperate for a leak as much with the omentum in place.

Dr Darling. You don't know that they would have leaked, though.

Dr Sepesi. It is impossible to state that an individual patient did not have an anastomotic leak purely because of the omental reinforcement. Based on the results of our study the number needed to treat is 17 patients; that is, 17 patients will need omental transfer to avoid 1 anastomotic leak. 
Dr Darling. You reported that surgeon was an independent predictor of leak rates. We and others have shown that there is a significant volume-outcome relationship for esophagectomy. This has generally been analyzed by center volume, not by surgeon volume. Do you think that individual surgeon volume is an important factor in outcomes after esophagectomy?

Dr Sepesi. I believe that an individual surgeon's volume is important to outcomes, but we have no evidence that this is driving the leak rate or the benefit from omentum reported by our group.

Dr Wayne Hofstetter (Houston, Tex). All surgeons at MD Anderson who participated in this study group are experienced and have a high volume. There are different operative techniques that go into each individual anastomosis. Some surgeons perform an anastomosis high up in the chest, some low in the chest; some form a large conduit, some a narrow conduit (4-cm wide vs $10-\mathrm{cm}$ wide). Those subtle differences cannot be accounted for in the data. We cannot go back and look retrospectively at those data. So in putting in a surgeon variable, we were basically using a surrogate marker to capture those individual techniques that go into the anastomosis.

Dr Stephen Cassivi (Rochester, Minn). I have a question on that very topic. Was the surgeon actually independently a factor other than the use of the omentum around the esophagus?

Dr Sepesi. Both surgeon and omentum were determined to be independent variables in the multivariate analysis.

Dr Nasser Altorki (New York, NY). I have 2 questions. First, your abstract says that minimally invasive esophagectomy (MIE) was a variable in the multivariate analysis, but your presentation doesn't show that.

Dr Sepesi. When we introduced the surgeon variable, the MIE variable was thrown out of the stepwise multivariate analysis. All variables with $P$ values $<.25$ on univariate analysis were included in the multivariate analysis. MIE was included in the analysis but was thrown out of the model. The only 2 remaining variables were surgeon and the omentum.

Dr Altorki. So you threw out all the neck anastomoses. Did any of the surgeons make a practice out of amputating the tip of the gastric conduit in the chest before performing the anastomosis?

Dr Sepesi. We do amputate the tip.

Dr Altorki. So why is that not a variable influencing the anastomotic leak rate in those patients who got omentum versus those who did not? In a heavily irradiated fundus, you cut off the tip and then you do the anastomosis further down in the conduit. That's 1 variable, and it may interact with the use of omentum.

Dr Thomas Waddell (Toronto, Ontario, Canada). I'm going to come back to that surgeon question again. Dr Hofstetter said that we can't possibly dissect out the issues, but this seems to be an attempt to do that. This is looking at different surgeons' practices and trying to identify what 1 surgeon does versus another. My question is, if you can't do that, what other programs can you consider to try and make the worst surgeon have the same leak rate as the best surgeon?

Dr Sepesi. Individual surgeons in our study noticed a significantly decreased leak rate when they started using the omentum. So a "high leak" surgeon may potentially decrease his or her leak rate with the use of omentum.

Dr Waddell. We just discussed the independence of this on multivariate analysis. That means that there are things other than using the omentum.

Dr Tomasz Grodzki (Szczecin, Poland). I have a short, technical question. The omentum in this case is supported by the same vessel (the gastroepiploic). Are you not afraid that it is less sufficient for the tip of the conduit if it needs to also support the omentum? In the case of failure, when the omentum was wrapped and the leak happened, what did you find during reoperation? Necrosis of the omentum? Insufficiency of the coverage?

Dr Sepesi. I think the only way to know about the differences in blood flow within the gastric conduit and the omentum is to do a perfusion study of the perforators versus the main artery. We have not done that. We have not noticed a compromised conduit because of the omental pedicle.

Dr Grodzki. In the case of failure, how did the omentum look?

Dr Sepesi. Except in the case of patients who lost the conduit, the omentum was viable. 